\title{
CP, T Violation
}

\section{José Bernabeu'}

Department of Theoretical Physics, University of Valencia,

IFIC, Joint Centre Univ. Valencia-CSIC, E-46100 Burjassot, Spain

E-mail: jose.bernabeuduv.es

The measured CP-Violation (CPV) asymmetries for Kaons and B's are well described by the Cabibbo-Kobayashi-Maskawa (CKM) Unitary Mixing Matrix Mechanism, but the current level of experimental accuracy and theoretical uncertainties leaves room for additional sources of $\mathrm{CPV}$, as demanded by Baryogenesis. I discuss Flavour-Changing-Neutral Current (FCNC) processes sensitive to Non-Decoupling Effects of New Physics. A direct Time-ReversalViolation (TRV) Effect was clearly observed in 2012 in the time evolution of neutral B-mesons. The conceptual basis to bypass the irreversibility of decays, and prepare in the B-Factories both the Reference and the T-reverse transitions, is provided by the quantum properties of Entanglement and the Decay as a Filtering Measurement. Flavour and CP eigenstate decay channels allow a separate independent measurement of CP, T and CPT asymmetries. Prospects for extending such a programme to any pair of B-decays and for Kaons in DAPHNE are discussed.

Proceedings of the Corfu Summer Institute 2014 "School and Workshops on Elementary Particle Physics and Gravity"

3-21 September 2014

Corfu, Greece

\section{${ }^{1}$ Speaker}




\section{Introduction}

In 2014 we have celebrated 50 years of CP-Violation (CPV). On July 10th 1964 Cronin, Fitch, Christenson and Turlay submitted a paper to Phys. Rev. Lett. "Evidence for the $\pi \pi$ decay of the $\mathrm{K}_{\mathrm{L}}$ meson" [1], announcing the discovery of CPV in the weak decays of neutral Kaons. For this discovery, the Nobel Prize in Physics 1980 was awarded to James Cronin and Val Fitch. The CP-Symmetry Breaking is understood as a consequence of the particle content in the Standard Model (SM). In 1973 Kobayashi and Maskawa published the paper "CP-Violation in the Renormalized Theory of Weak Interaction" in Progress of Theoretical Physics [2] and they shared one half of the Nobel Prize in Physics 2008 "for the discovery of the origin of the broken symmetry which predicts the existence of at least three families of quarks in nature". The direct evidence of Time-Reversal-Violation (TRV) was clearly established in the time evolution of neutral B-mesons by the BABAR Collaboration and in 2012 the paper "Observation of TimeReversal Violation in the $\mathrm{B}^{0}$ Meson System" was published in Phys. Rev. Lett. [3], following the lines of the conceptual basis for entangled neutral mesons in the B-factories discussed in the paper [4] "CP, T and CPT versus temporal asymmetries for entangled states of the $\mathrm{B}_{\mathrm{d}}$-system".

In Section 2 we discuss the fact that CPV asymmetries are well described by the CabibboKobayashi-Maskawa Quark Mixing Matrix Mechanism, but the current level of experimental accuracy and theoretical uncertainties leaves room for additional sources of CPV, as required by Baryogenesis in the Universe. We identify potential transitions in B-physics, using FlavourChanging-Neutral. Current (FCNC) processes and CPV asymmetries, able to incorporate virtual contributions of New Physics through Non-Decoupling Effects. Section 3 is devoted to TRV concepts and results: what is "Time Reversal" in classical and quantum mechanics, the NO-GO argument for its search with unstable particles, its By-pass using Entanglement and the Decays as Filtering Measurements for entangled $B^{0}-\bar{B}^{0}$ system in the B-Factories, and $K^{0}-\bar{K}^{0}$ system in the $\Phi$-Factory, the role of time-ordered decay channels to Flavour and CP-eigenstates for disentangling genuine separate independent asymmetries for CP, T and CPT and the $14 \sigma$ observation of TRV by the BABAR experiment. Finally, Section 4 presents our results, conclusions and prospects

\section{CP Violation}

It is well known since 1957 that weak interactions have little respect for symmetries. That year space inversion (parity, P) symmetry was discovered to be broken in $\beta$ decays [5-7]. Then, there was the hope that the combination of $\mathrm{P}$ with charge conjugation (CP) was a good symmetry. But just a few years later, in 1964, there was discovered a small but unambiguous violation of the CP symmetry in $\mathrm{K}$ meson decays [1,8]. More recently, in 2001, the B factory experiments BABAR and Belle, observed that $\mathrm{CP}$ is violated in $\mathrm{B}$ mesons $[9,10]$.

The now well established $\mathrm{CP}$ violation in the quark sector can be successfully accommodated within the Standard Model (SM) of particles and fields through the three-family Cabibbo-Kobayashi-Maskawa (CKM) quark-mixing mechanism [11,2]. It describes the coupling of the $\mathrm{W}$ boson to up and down quarks and conveys the fact that the quarks with definite properties under charged-current weak interactions are linear combinations of the quark mass eigenstates [12]. For three families, the unitarity conditions of the quark-mixing matrix $\mathrm{V}$ are represented by triangles in the complex plane, as illustrated in figure 1, and lead to four fundamental parameters: three magnitudes and one single irreducible phase. 


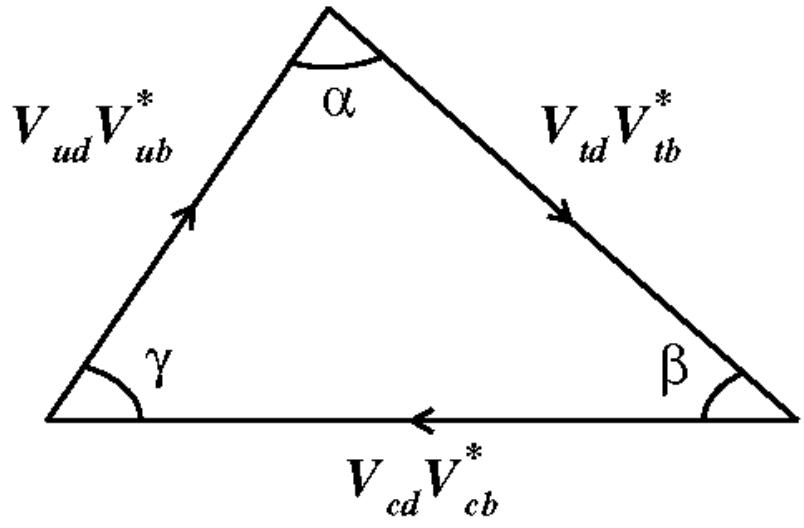

Figure 1. The bd unitarity triangle representing the CKM unitarity conditions. The three sides are determined from semileptonic and non-leptonic $\mathrm{B}$ decays, including $B^{0}-\bar{B}^{0}$ oscillations. Since they are of comparable length the angles are sizeable and one expects large $\mathrm{CP}$ asymmetries in B decays in the SM. There are other two triangles which almost collapse to a line. This gives an intuitive understanding of why $\mathrm{CP}$ violation is small in the leading $\mathrm{K}$ decays (ds triangle) and in the leading $\mathrm{B}_{\mathrm{s}}$ decays (bs triangle).

In the Wolfenstein parameterization [13] we can write $\mathrm{V}$ to $\mathrm{O}\left(\lambda_{c}^{4}\right)$ as

$$
\mathrm{V}=\left[\begin{array}{ccc}
1-\lambda_{c}^{2} / 2 & \lambda_{c} & A \lambda_{c}^{3}(\rho-i \eta \\
-\lambda_{c} & 1-\lambda_{c}^{2} / 2 & A \lambda_{c}^{2} \\
A \lambda_{c}^{3}(1-\rho-i \eta) & -A \lambda_{c}^{2} & 1
\end{array}\right]
$$

where the matrix line runs over $\mathrm{d}, \mathrm{s}$, and $\mathrm{b}$ quarks, and $\lambda_{\mathrm{c}} \approx 0.226, \mathrm{~A} \approx 0.814, \rho \approx 0.135$ and $\eta \approx$ 0.34931 .

Extensive tests of the CKM mechanism using all experimental data show a high degree of consistency [14]. Historically, Kobayashi and Maskawa extended in 1973 the $2 \times 2$ Cabibbo mixing matrix to $3 \times 3$ to explain the $\mathrm{CP}$-violation discovered nine years before, thus anticipating the existence of the third family of quarks, quickly confirmed with the discovery of the $\tau$ lepton in 1975[15] and of the fifth quark, the b, two years later [16].

At the asymmetric B factories, electron and positron beams collide with high luminosity at a c.m. energy of $10.58 \mathrm{GeV}$ corresponding to the mass of the $\mathrm{Y}(4 \mathrm{~S})$ resonance, a vector particle with $\mathrm{J}^{\mathrm{PC}}=1--$. The $\mathrm{Y}(4 \mathrm{~S})$ is a bound state of $\mathrm{a} \mathrm{b}$ and $\mathrm{a} \bar{b}$ quark, that decays exclusively to a pair of $\mathrm{B}$ and $\bar{B}$ mesons. Since the mass of the Y(4S) is only slightly higher than twice the mass of the $\mathrm{B}$ meson, the two $\mathrm{B}$ mesons have low momenta (about $330 \mathrm{MeV} / \mathrm{c}$ ) and are produced almost at rest in the $\mathrm{Y}(4 \mathrm{~S})$ reference frame with no additional particles besides those associated to the $\mathrm{B}$ decays. The energy of the electron beam is adjusted to be between twice and three times larger than that of the positrons, so that the c.m. frame has a Lorentz boost along the collision axis. Two B factory colliders, PEP-II at SLAC in California and KEKB at KEK in Japan, with their corresponding detectors, BABAR [17,18] and Belle [19], have been operating during the last decade, accumulating an integrated luminosity of data exceeding $500 \mathrm{fb}^{-1}$ and $1 \mathrm{ab}^{-1}$, respectively. To the B factory programme we add in the last few years the LHCb experiment at CERN [20] with new and complementary information about rare decays, FCNC processes and CP Violation.

For the $\mathrm{B}_{\mathrm{d}}$ system CPV in the mixing is very small. The interference of mixing and nomixing amplitudes in the $\mathrm{B}^{0}$ decays is observed in decay products which are accessible by both $\mathrm{B}^{0}$ and $\bar{B}^{0}$. The corresponding CPV asymmetry can be written as

$$
\mathrm{A}_{f}(\mathrm{t})=\mathrm{S}_{f} \sin (\Delta \mathrm{mt})-\mathrm{C}_{f} \cos (\Delta \mathrm{mt})
$$

where $\Delta \Gamma=0$ is assumed. The most precise asymmetries are measured in the tree-dominated $\mathrm{b}$ $\rightarrow$ ccs transitions, such as $\mathrm{B}^{0} \rightarrow \psi \mathrm{K}^{0}$, and are given by [21] (we use $\mathrm{K}^{0}$ throughout to denote results that combine $\mathrm{K}_{\mathrm{S}}$ and $\mathrm{K}_{\mathrm{L}}$ modes): $\mathrm{S}\left(\psi \mathrm{K}^{0}\right)=+0.682 \pm 0.019$. The penguin contributions are very small, so that one has the interpretation in the $\mathrm{SM} S=\sin (2 \beta), \mathrm{C}=0$. 
$\mathrm{CP}$ violation in the interference of mixing and decay in the $\mathrm{B}^{0} \rightarrow \pi^{+} \pi^{-}$mode is given by [21] $\mathrm{S} \pi^{+} \pi^{-}=-0.66 \pm 0.06$. It is interpreted in the $\mathrm{SM}$ as $\mathrm{S}=\sin (2 \alpha)$.

For the $\mathrm{B}^{0}$ system, the phase of the mixing amplitude is determined from the intermediate top-quark exchange in the box diagram, so that the SM interpretations of these interferences in terms of the $\beta$ and $\alpha \mathrm{CP}$ angles of the unitarity triangle are apparent. On the contrary, the phase $\gamma$ in the unitarity triangle involves the interference of the sides for decays with charm and up quark constituents, without any relation to the mixing. The $\mathrm{CP}$ angle $\gamma$ is thus a measure of Direct CPV. Its measurement has been undertaken by BABAR, BELLE and LHCb with the decay $\mathrm{B}^{+} \rightarrow \mathrm{D} \mathrm{K}^{+}$and other related transitions. The extraction of $\gamma$ needs a detailed analysis involving in addition the presence of strong phases associated to final-state hadronic interactions. The present average value is [21] $\gamma=(67 \pm 12)^{0}$. An ideal experiment would be one in B factories using Entanglement and detecting the pair of decays $\rightarrow \psi \mathrm{K}^{0}$ and $\rightarrow \pi \pi$ at equal times without any effect of the mixing phase. It remains to be seen whether this gedanken experiment can become a real experiment in the upgraded SuperBELLE.

The rare decay $\mathrm{B}_{\mathrm{s}} \rightarrow \mu \mu$ has been observed by LHCb and CMS. Based on the presence of a FCNC penguin amplitude induced by Z-exchange as seen in the diagram

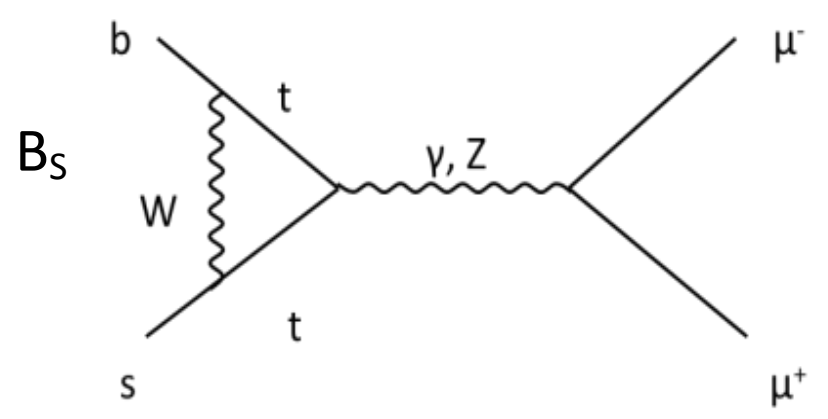

one could expect a priori New Physics virtual effects induced by Non-Decoupling of longitudinal contributions. The result [22] presented in figure 2 has represented the latest disappointment of the scientific community.

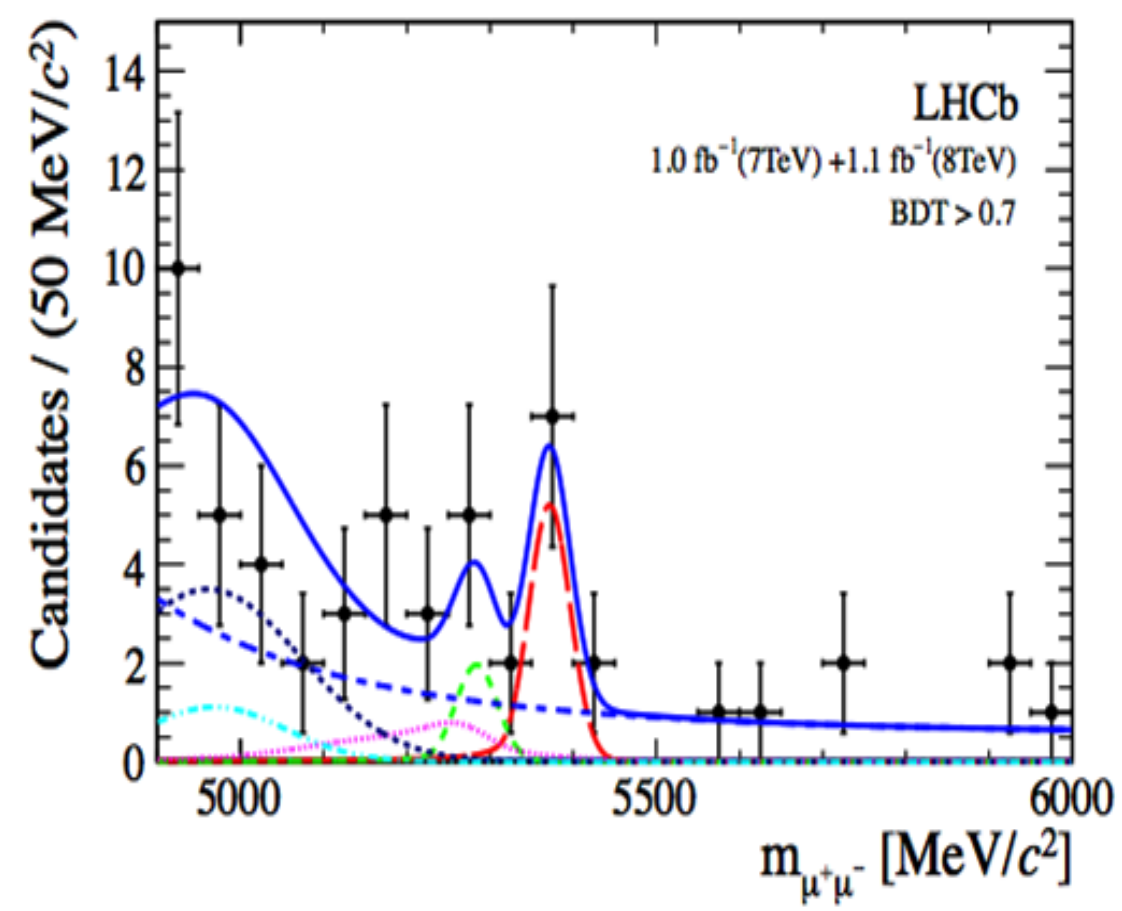

Figure 2. The invariant mass distribution of the $\mu \mu$ system showing the Bs-peak. 
The experimental Branching Ratio is given by

$$
B\left(B_{s}^{0} \rightarrow \mu^{+} \mu^{-}\right)=\left(3.2_{-1.2}^{+1.4}(\text { stat })_{-0.3}^{+0.5}(\text { syst })\right) \times 10^{-9}
$$

in perfect agreement with the SM value $(3.23 \pm 0.27) \times 10^{-9}$. In some models, like SuperSymmetry, the result could have been orders of magnitude different from the SM value. One has to be aware, however, that in this process the FCNC penguin is projected to a pseudoscalar, so that its contraction with the leptonic vertex leads to helicity suppression and an amplitude proportional to the mass of the lepton. The FCNC b-s penguin vertex can be probed under more general conditions by the opening of the b,s lines in the decays $Z \rightarrow b \bar{s}(\bar{d})$ at the $\mathrm{Z}$ peak [23] using the high number of Z's produced at LHC. Another way is by searching for the processes B $\rightarrow$ $\mathrm{K}\left(\mathrm{K}^{*}\right) 1^{+} 1^{-}$, which open new effective current operators in scalar-scalar and scalar-vector matrix elements. In fact, the process has been observed and the analysis, using the experimental results of $\mathrm{LHCb}$, making use of the OPE formalism leads to intriguing tensions with the SM expectations in some of the effective operators [24]. If these discrepancies are associated to longitudinal amplitudes of the mediators, one should seriously consider the search of the process $B \rightarrow K\left(K^{*}\right)$ $v v$ in order to avoid the amplitudes mediated by $\gamma$-exchange. As mentioned before, the hadronic vertex for these transitions leads to an amplitude which is not proportional to the neutrino mass.

Non-Decoupling from longitudinal contributions which are not giving an helicity-suppressed amplitude can also be obtained from the process $\mathrm{B}_{\mathrm{s}} \rightarrow v v \gamma$, as shown in the diagram

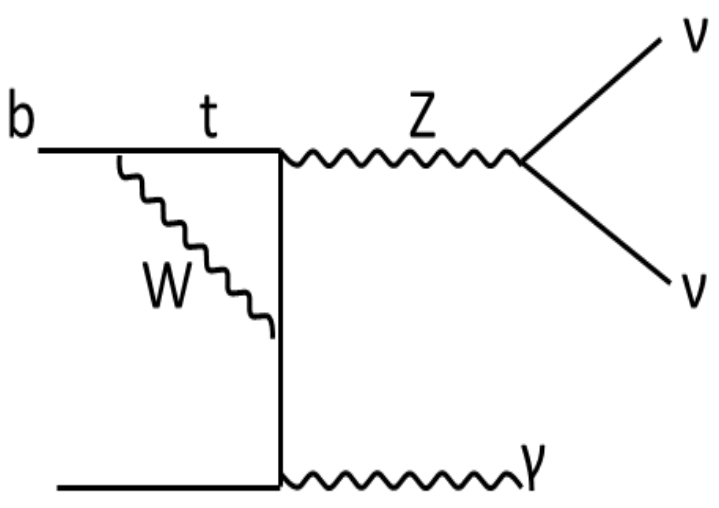

An existing upper limit [25] by BABAR gives $\mathrm{Br}<4.7 \times 10^{-5}, 90 \%$ C.L.

\section{Time-Reversal Violation}

The symmetry transformation that changes a physical system with a given sense of the time evolution into another with the opposite sense is called Time-Reversal T. It corresponds to changing the sign of the velocity vector $\mathbf{v}$ or the momentum $\mathbf{p}$, without changing the position $\mathbf{r}$. In the dynamical equations of motions, or their solutions, such a transformation corresponds formally to replacing $t$ by $-t$. The $T$ transformation changes the sign of other dynamical variables such as angular momentum. For fields, the magnetic field changes its sign under time reversal, whereas the electric field does not.

The time-reversal transformation in classical mechanics corresponds to substitute for each trajectory $\mathbf{r}(\mathrm{t})$ the trajectory $\mathbf{r}(-\mathrm{t})$, i.e. to moving along the given trajectory with the opposite velocity at each point, as illustrated in figure 3. It is not obvious that the dynamics remains invariant under this $\mathrm{T}$ transformation. If the original trajectory is dynamically possible, $\mathrm{d} \mathbf{p} / \mathrm{dt}=$ $\mathbf{F}$ with a force $\mathbf{F}$ depending on the sense (sign) of the velocity leads to a violation of $\mathrm{T}$ invariance. This observed violation would be a fake violation if there is an external agent which is not T-invariant, like friction in general or a magnetic field for charged particles. 


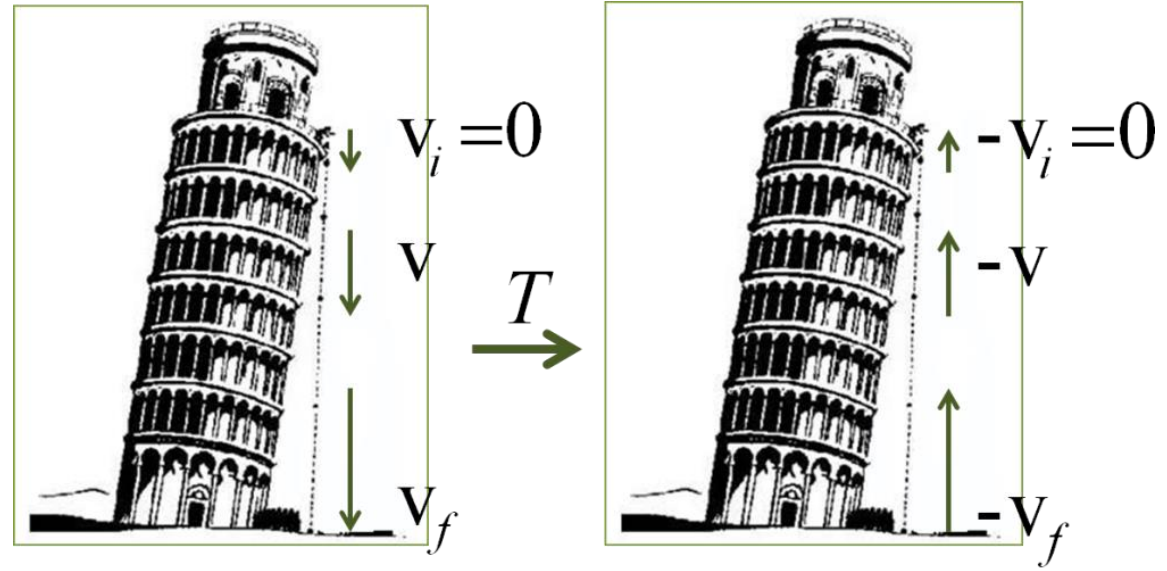

Figure 3. (a) Trajectory of a stone falling from the leaning tower.

(b) Trajectory after time-reversal transformation.

In quantum mechanics, Wigner's time-reversal transformation [26],

$$
\psi(t) \rightarrow T \psi(t) \equiv \psi_{T}(t)=\psi^{*}(-t)
$$

keeps the Schrodinger equation, i $\hbar \psi(\mathrm{t}) / \partial \mathrm{t}=\mathrm{H} \psi(\mathrm{t})$, invariant under a $\mathrm{T}$ transformation if the Hamiltonian $\mathrm{H}$ is real. This has three fundamental consequences:

First, the T operator is antiunitary. This property can be seen, for example, evaluating the scalar product of two states,

$$
\left\langle\psi_{T}(t) \mid \phi_{T}(t)\right\rangle=\langle\psi(-t) \mid \phi(-t)\rangle^{*}=\langle\phi(-t) \mid \psi(-t)\rangle
$$

Thus, time reversal has to do with interchange of bra and ket states. Second, the complex conjugation implies that time reversal does not have observable and conserved eigenvalues. Third, for a plane wave with momentum $\mathbf{p}, \psi(\mathbf{r} ; \mathrm{t})=\exp [i(\mathbf{p} \cdot \mathbf{r}-\mathrm{Et}) / \hbar]$, the time-reverse wavefunction is $\psi^{*}(\mathbf{r},-\mathrm{t})=\exp [\mathrm{i}(-\mathbf{p} \cdot \mathbf{r}-\mathrm{Et}) / \hbar \mathrm{h}]$, i.e. the T-transformed function describes a particle with momentum $-\mathbf{p}$ and energy $\mathrm{E}$, thus it is not necessary to interpret the transformed function as a particle going backwards in time. For this reason the $\mathrm{T}$ transformation is often referred to as "motion reversal" rather than "time reversal".

The $\mathrm{T}$ transformation is implemented in the space of states by the antiunitary operator $U_{T}$ in such a way that, for spinless particles,

$$
U_{T} \boldsymbol{r} U_{T}^{\dagger}=\boldsymbol{r}, \quad U_{T} \boldsymbol{p} U_{T}^{\dagger}=-\boldsymbol{p}
$$

and $\psi_{\mathrm{T}}(\mathrm{t})=U_{T} \psi(-\mathrm{t})$. Equation (5) guarantees the invariance of the commutation rule between $\mathbf{r}$ and $\mathbf{p}$, thus we might say that $\mathrm{T}$ transforms quantum mechanics into quantum mechanics. For a Hamiltonian $\mathrm{H}$ invariant under time reversal, $\left[\mathrm{H} ; U_{T}\right]=0$, the time-evolution operator $\mathcal{U}\left(\mathrm{t} ; \mathrm{t}_{0}\right)$ transforms as

$$
U_{T} \mathcal{U}\left(t, t_{0}\right) U_{T}^{\dagger}=\mathcal{U}^{\dagger}\left(t, t_{0}\right)
$$

The antiunitary character of $U_{T}$ allows to write $U_{T}=\mathrm{UK}$, where $U$ is unitary $\left(U^{I}=U^{\dagger}\right)$ and $\mathrm{K}$ is an operator which complex conjugates all complex numbers. For the matrix elements of time-dependent transitions we have

$$
\begin{gathered}
\left\langle f\left|\mathcal{U}\left(t, t_{0}\right)\right| i\right\rangle=\left\langle f\left|U_{T}^{\dagger} U_{T} \mathcal{U}\left(t, t_{0}\right) U_{T}^{\dagger} U_{T}\right| i\right\rangle \\
=\left\langle U_{T} f\left|\mathcal{U}^{\dagger}\left(t, t_{0}\right)\right| U_{T} i\right\rangle^{*}=\left\langle U_{T} i\left|\mathcal{U}\left(t, t_{0}\right)\right| U_{T} f\right\rangle
\end{gathered}
$$

where time-reversal invariance is assumed in equation (7). As a consequence, the comparison between $\mathrm{i} \rightarrow \mathrm{f}$ and $U_{T} \mathrm{f} \rightarrow U_{T}$ i transitions is a genuine test of this invariance. It is because of 
these special properties that the role of time reversal is distinct from that of any other symmetry operation in physics, and makes its experimental investigation significantly more difficult than other symmetries.

A direct consequence of quantum dynamics is the negative-exponential time behavior of the decay of any unstable system into two or more particles, as given by the Fermi golden rule. The reversal of the exponential decay law reveals that the $\mathrm{T}$ transformation is not defined for a decaying state [27], thus it appears that the decay prevents proofs of motion reversal. The decay is irreversible, and it behaves like the arrow of time explained by the increase of entropy. The TRV asymmetry should not contain the decay as an essential ingredient.

There are two main types of experiments or observables that can be used to detect directly time-reversal non-invariance [28-30]. First, a non-zero expectation value of a T-odd operator for a non-degenerate stationary state. This is the case for an electric dipole moment (e.d.m.) of a particle with spin, which is also a P-odd, C-even quantity. Thus, if either parity or time reversal are good symmetries, the particle cannot have an e.d.m. A non-zero e.d.m. can be generated by either strong interaction T violation, unless it is annulled by a Peccei-Quinn symmetry leaving the axion as remnant [31], or by T violation in weak interactions. In the SM with the CKM mechanism, a non-vanishing e.d.m. of the neutron only appears at three-loop level. Hence, these experiments probe for physics beyond the SM. To date, no signals for e.d.m. have been found, although there are strong limits, as for the neutron and the electron, $\left|d_{n}\right|=2.9 \times 10^{-26} e-$ cm and $\left|d_{e}\right|=1.05 \times 10^{-27} e-c m[32]$.

Second: in transitions, with the requirement of exchanging initial and final states to compare the T-reverse transition with the Reference transition. We might consider this exchange in the mixing of the pseudoscalar neutral $\mathrm{K}, \mathrm{B}$ and $\mathrm{D}$ mesons. In this case one compares the probability of a flavor eigenstate (say) $\mathrm{K}^{0}$ transforming into a $\bar{K}^{0}$, and viceversa. Since the states $\mathrm{K}^{0}$ and $\bar{K}^{0}$ are particle and antiparticle, the two transitions are connected by both T and CP transformations. Even if CPT symmetry would be broken, there exists no difference between CP and $\mathrm{T}$ in this case. Thus the two symmetry transformations are experimentally identical and lead to the same asymmetry. This flavor-mixing or Kabir asymmetry [33] is independent of time since the two processes have identical time dependence, and it is induced by the interference between the dispersive, $\mathrm{M}_{12}$, and absorptive, $\Gamma_{12}$, contributions in the effective Hamiltonian. Evidence at $4 \sigma$ level for this asymmetry was found by the CPLEAR experiment [34] at CERN in 1998 for $\mathrm{K}^{0} \rightarrow \bar{K}^{0}$ in $\bar{p}-p$ collisions. The strangeness (strange and antistrange flavor content) of the $\mathrm{K}^{0}$ and $\bar{K}^{0}$ mesons at production time was determined by the charge of the accompanying charged kaon. Since weak interactions do not conserve strangeness, the $\mathrm{K}^{0}$ and $\bar{K}^{0}$ may subsequently transform into each other. The strangeness of the neutral kaon at decay time is determined through the semileptonic decay $\mathrm{K}^{0} \rightarrow \mathrm{e}^{+} \pi^{-} v$. The asymmetry, shown in figure 4 , is effectively independent of time, i.e., not built in the time evolution, and reveals a net value with respect to zero.

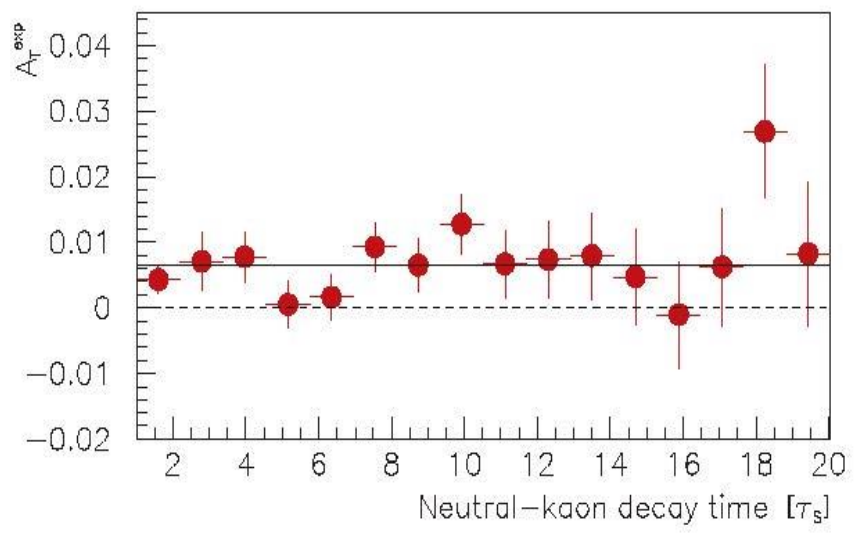

Figure 4. The CPLEAR asymmetry versus the neutralKaon decay time

(in units of the KS lifetime) [34].

The solid line represents the average. 
The interpretation of the asymmetry relies on two main aspects. Firstly, in the framework of the Weisskopf-Wigner approach $[35,36]$, the effect comes from the overlap (non-orthogonality) of the "stationary" $\mathrm{K}_{\mathrm{S}}$ and $\mathrm{K}_{\mathrm{L}}$ states of definite mass and lifetime. Secondly, the decay plays an essential role; indeed, the dispersive and absorptive contributions to $K-\bar{K}^{0}$ mixing are at leading order proportional to the mass and decay width differences between $\mathrm{K}_{\mathrm{L}}$ and $\mathrm{K}_{\mathrm{S}}$, respectively. The presence of the decay as an initial state interaction, essential to construct a non-vanishing interference for this observable, has been argued by Wolfenstein to claim that this asymmetry "is not as direct a test of time-reversal violation as one might like" [27,28]. In the neutral Bsystem, where the decay width difference between the $\mathrm{B}^{0}$ mass eigenstates is negligible, the measurement of the asymmetry has, in fact, brought negative results [37,38]. Other authors, however, have argued that its interpretation as a genuine signal for T-violation does not get affected by these arguments [39-41].

A direct evidence for TRV would mean an experiment which, by itself, is able to establish a non-vanishing genuine TRV asymmetry independent of CPV or CPT invariance. The problem is then the filtering of definite initial and final states of the neutral meson for the Reference and Treverse transitions.

The solution $[4,42-45]$ arises from the quantum mechanical properties imposed by the EPR entanglement $[46,47]$ between the two neutral B mesons produced in the $\gamma(4 \mathrm{~S})$ resonance decay. As the individual state of each meson in the entangled system is not defined before the first decay, just as one $\mathrm{B}$ meson in the entangled pair is prepared in the definite flavour $B^{0}$ or $\bar{B}^{0}$ states at the time when the other $B$ is projected into its orthogonal by the observation of a semileptonic decay, the first decay of one B into the definite CP final states $\mathrm{J} / \psi \mathrm{K}_{\mathrm{S}}$ or $\mathrm{J} / \psi \mathrm{K}_{\mathrm{L}}$ prepares the other living B into well defined, orthogonal linear combinations $B_{-}$or $B_{+}$of $B^{0}$ and $\bar{B}^{0}$ states. In fact, this idea offers the opportunity to explore separately time reversal T, CP and CPT asymmetries, selecting appropriately different transitions defined by different decay channels.

The entangled state of the two mesons produced by the $\Upsilon(4 S)$ decay can be written in terms of any pair of orthogonal states of the individual B mesons as

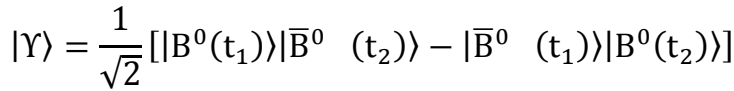

$$
\begin{aligned}
& =\frac{1}{\sqrt{2}}\left[\left|\mathrm{~B}_{+}\left(\mathrm{t}_{1}\right)\right\rangle\left|\mathrm{B}_{-}\left(\mathrm{t}_{2}\right)\right\rangle-\left|\mathrm{B}_{-}\left(\mathrm{t}_{1}\right)\right\rangle\left|\mathrm{B}_{+}\left(\mathrm{t}_{2}\right)\right\rangle\right]
\end{aligned}
$$

Its time evolution, including mixing, keeps only, by virtue of the antisymmetry, terms $B^{0}, \bar{B}^{0}$ or $\mathrm{B}_{+}$, $\mathrm{B}_{\text {. }}$. What is T-transformation experimentally? As illustrated in figure 5, suppose that the Reference transition is defined by the time-ordered decay channels $1^{+}$first, $\mathrm{J} / \psi \mathrm{K}_{\mathrm{S}}$ later, as shown in the left-hand side of figure 5. The use of Entanglement plus the Decay as a Filtering Measurement tells us that the meson transition corresponds to $\bar{B}^{0} \rightarrow B_{-}$. In terms of meson states, the T-reverse transition is then $B_{-} \rightarrow \bar{B}^{0}$ and the question arises: Which are the timeordered decay channels which correspond to this T-reverse transition? For definite flavour and $\mathrm{CP}$ eigenstates, orthogonality of $B^{0}, \bar{B}^{0}$ and $\mathrm{B}_{+}, \mathrm{B}_{\text {- provides the solution: } \mathrm{J} / \psi \mathrm{K}}$ first, $1^{-}$later. 

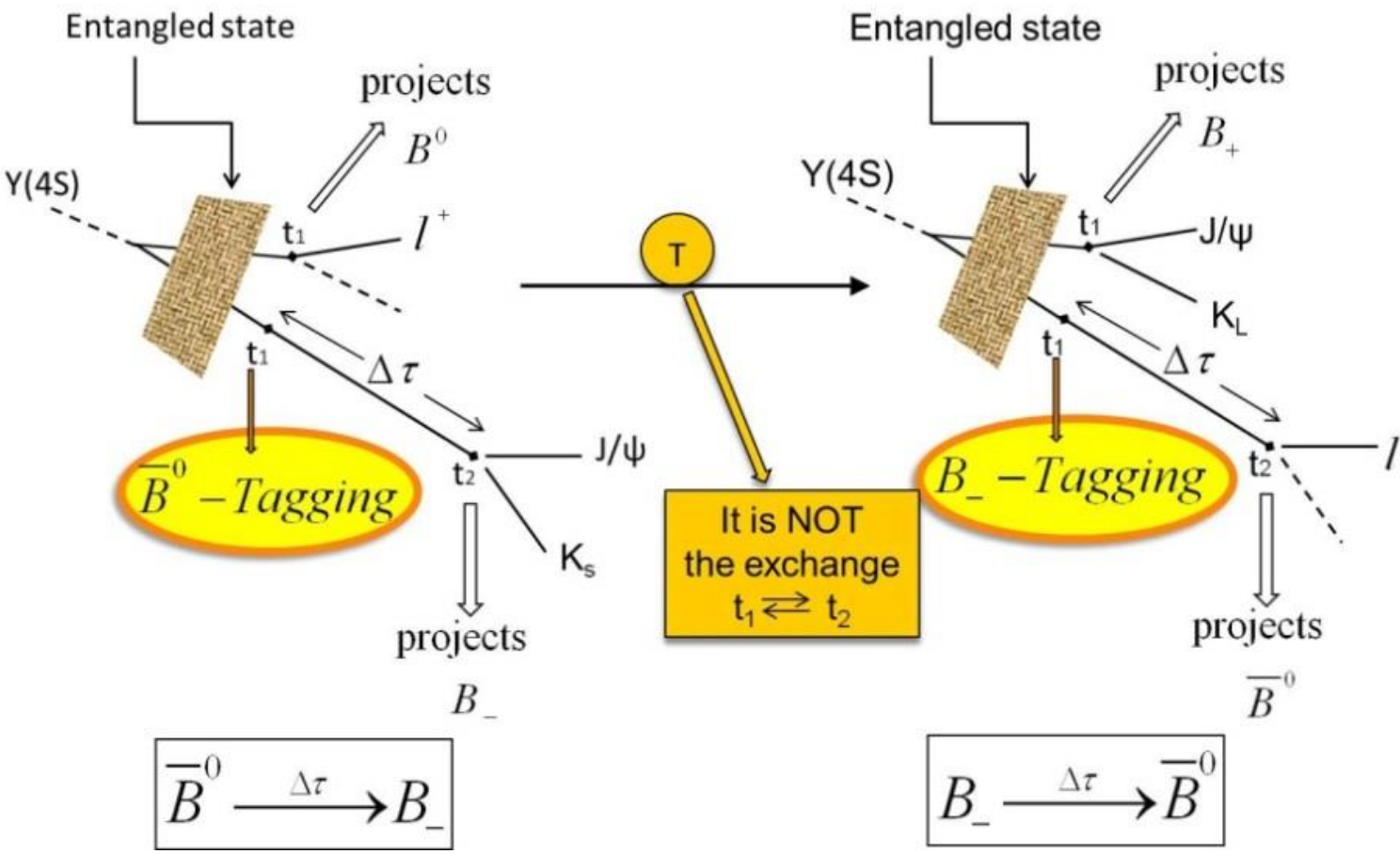

Figure 5. Basic concepts explaining the preparation and detection of initial and final meson states, for the Reference and T-reverse transitions, by means of the experimental time-ordered decay channels to definite flavour and CP eigenstates.

There are eight processes of this kind: 2 for flavour $\mathrm{x} 2$ for CP $\mathrm{x} 2$ time-orderings. Each of them has a time dependent probability distribution of the following form

$$
I_{i}(\Delta t) \sim e^{-\Gamma \Delta t}\left\{C_{i} \cos (\Delta m \Delta t)+S_{i} \sin (\Delta m \Delta t)\right\}
$$

with the only use of quantum mechanics, and $\Delta \Gamma=0$ for the neutral $\mathrm{Bd}$ mesons.

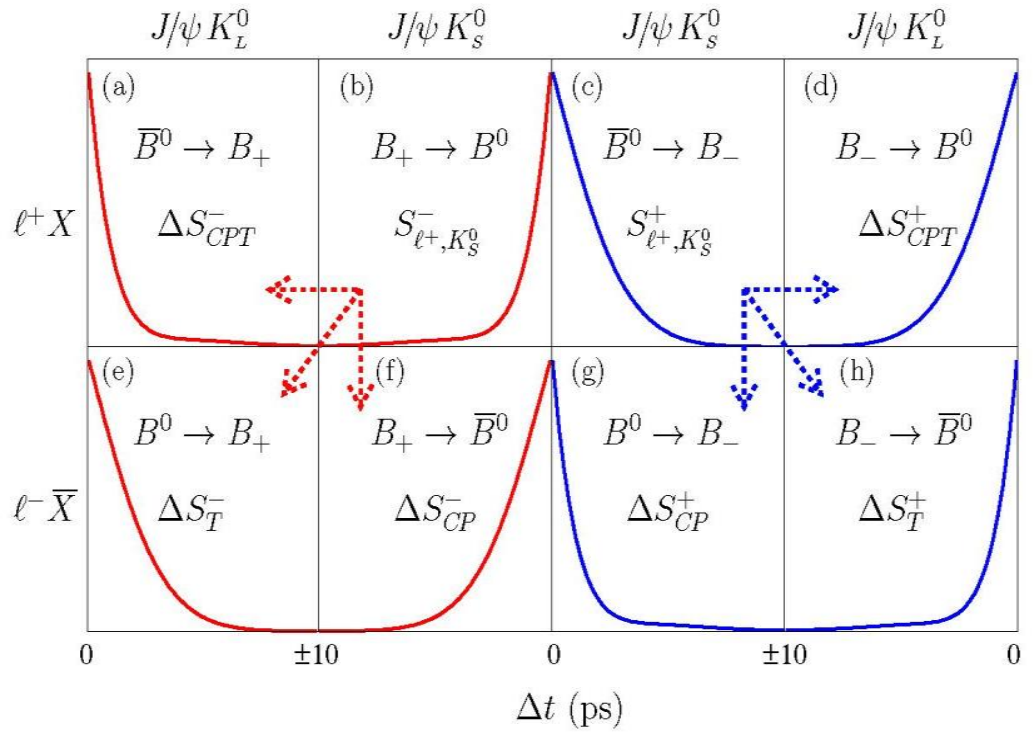

Figure 6. Timedependent probability distributions for the eight timeordered event classes associated to flavourCP eigenstate decay channels, shown as a planar map. The independent $\mathrm{CP}, \mathrm{T}$ and CPT asymmetries from a given Reference transition are apparent. 
BABAR reported in November 2012 the measurement [3] of these eight time-ordered event classes. From the eight pairs $(\mathrm{S}, \mathrm{C})$ of signal coefficients, one might construct two sets of three pairs each of independent asymmetry parameters, $\left(\Delta S_{T}^{ \pm} ; \Delta C_{T}^{ \pm}\right),\left(\Delta S_{C P}^{ \pm} ; \Delta C_{C P}^{ \pm}\right)$and $\left(\Delta S_{C P T}^{ \pm} ; \Delta C_{C P T}^{ \pm}\right)$. They are given in the left panel of Table 1,

Table 1.

\begin{tabular}{l|r|c}
\hline Parameter & \multicolumn{1}{|c}{ Final result } & SM expected val. \\
\hline \hline$\Delta S_{T}^{+}$ & $-1.37 \pm 0.14 \pm 0.06$ & -1.36 \\
$\Delta S_{T}^{-}$ & $1.17 \pm 0.18 \pm 0.11$ & 1.36 \\
$\Delta C_{T}^{+}$ & $0.10 \pm 0.14 \pm 0.08$ & 0. \\
$\Delta C_{T}^{-}$ & $0.04 \pm 0.14 \pm 0.08$ & 0. \\
\hline$\Delta S_{C P}^{+}$ & $-1.30 \pm 0.11 \pm 0.07$ & -1.36 \\
$\Delta S_{C P}^{-}$ & $1.33 \pm 0.12 \pm 0.06$ & 1.36 \\
$\Delta C_{C P}^{+}$ & $0.07 \pm 0.09 \pm 0.03$ & 0. \\
$\Delta C_{T}^{-}$ & $0.08 \pm 0.10 \pm 0.04$ & 0. \\
\hline$\Delta S_{C P T}^{+}$ & $0.16 \pm 0.21 \pm 0.09$ & 0. \\
$\Delta S_{C P T}^{-}$ & $-0.03 \pm 0.13 \pm 0.06$ & 0. \\
$\Delta C_{C P T}^{+}$ & $0.14 \pm 0.15 \pm 0.07$ & 0. \\
$\Delta C_{C P T}^{-}$ & $0.03 \pm 0.12 \pm 0.08$ & 0. \\
\hline$S_{\ell^{+}, K_{S}^{0}}^{+}$ & $0.55 \pm 0.09 \pm 0.06$ & 0.68 \\
$S_{\ell^{+}, K_{S}^{0}}^{-}$ & $-0.66 \pm 0.06 \pm 0.04$ & -0.68 \\
$C_{\ell^{+}, K_{S}^{0}}^{+}$ & $0.01 \pm 0.07 \pm 0.05$ & 0. \\
$C_{\ell^{+}, K_{S}^{0}}^{-}$ & $-0.05 \pm 0.06 \pm 0.03$ & 0. \\
\hline
\end{tabular}

The Reference transitions are taken by convention $B_{+} \rightarrow B^{0}$ and $B^{0} \rightarrow B_{-}$. This choice has the advantage that the breaking of time-reversal symmetry would directly manifest itself through any nonzero value of $\Delta S_{T}^{ \pm}$or any difference between $\Delta S_{C P}^{ \pm}$and $\Delta S_{C P T}^{ \pm}$. It follows that the experimental results given in Table 1 demonstrate an unambiguous, direct evidence of timereversal violation in the time evolution of neutral B mesons, obtained through motion reversal in transitions that are not CP conjugate to each other. The reported TRV asymmetries do not need the decay as an essential ingredient and originate in the interference between mixing and nomixing amplitudes in the time evolution of the system. The asymmetry parameters $\Delta S_{T}^{ \pm}$are independent of the value of $\Delta \Gamma$.

The experimental results are, on the other hand, compatible with all C-coefficients vanishing. This a proof that the decay amplitudes have the same absolute values for particle and antiparticle, implying a single weak phase and no direct CP-violation in the decay. A recent analysis [48] of the conditions under which the measured motion reversal asymmetry is a true TRV effect reinforces the adequacy of the method. The comparison of the experimental results with the right-handed panel of Table 1 tells us the ability of the SM for reproducing all observations.

The four TRV independent raw symmetries measured by the BABAR experiment [3] are given in figure 7 


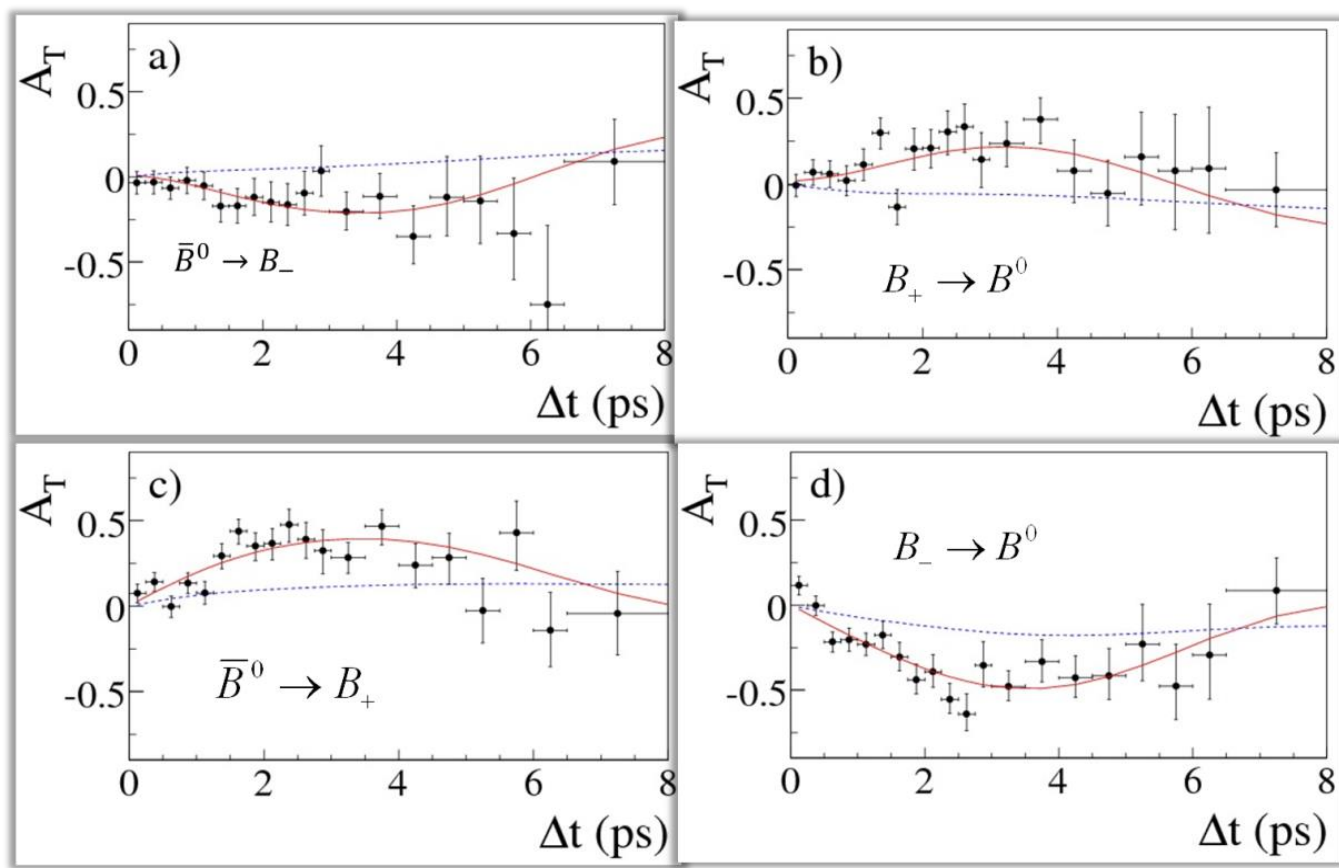

Figure 7. Experimental TRV asymmetries as function of $\Delta t$

They correspond to the asymmetries between the transitions $\bar{B}^{0} \rightarrow B_{-}, B_{+} \rightarrow B^{0}, \bar{B}^{0} \rightarrow B_{+}, B_{-} \rightarrow$ $B^{0}$, and their T-reverse transitions. The $\Delta$ t dependence demonstrates that the TRV effect is built during the time evolution of the neutral $\mathrm{B}$. The combined significance of the four measured nonvanishing asymmetries provides a conclusive $14 \sigma \mathrm{TRV}$ effect.

This discovery was made possible thanks to the spectacular quantum properties of the Einstein-Podolsky-Rosen entangled state. In the epistemological language for the nature of Reality one could say that "The reality of two entangled neutral B's is much more than the sum of the two separate neutral B-meson local realities". The appropriate preparation of initial and final meson estates, as required by a genuine measurement of a TRV effect, is based on: 1) Entanglement to transfer the information from the first decaying meson to its living partner; 2) the Decay as a Filtering Measurement for the meson state.

The conceptual basis which is at the origin of the TRV effect for neutral B mesons has been adapted [49] to the treatment of entangled neutral Kaons at the $\phi$-factory DAPHNE. The expectations for a measurement of the corresponding asymmetries at the upgraded facility have been calculated. Like for B's, one considers the pair of decay channels to definite Flavour and CP eigestate decay products for the preparation of the appropriate neutral Kaon states. The question arises: Can the method be extended to any pair of decay channels? There is an "orthoganility problem" for identifying which are the decay channels associated to the T-reverse transition when the decay products are no longer either Flavour or CP eigenstates. In order to bypass this problem, there is a recent suggestion [50] of taking a modified Reference meson transition from the detected pair of decay channels.

\section{Conclusions}

- CPV violating asymmetries are well described up to now by the SM mechanism of the CKM Quark Mixing Matrix. It is of highest importance the search of virtual effects of New Physics in rare decays that are sensitive to the details of the FCNC transitions and CPV, like in penguin vertices. The current level of experimental accuracy and theoretical uncertainties leaves room for additional sources of CPV. The motivation for new physics contributions includes the fact that the SM is unable to explain the Matter-Antimatter asymmetry of the Universe.

- The arguments used to preclude a true search of TRV in unstable particles have been by-passed by the transfer of quantum information, implied by Entanglement of the pair of me- 
sons in meson factories, from the decaying particle to its living partner. In these entangled systems, the preparation of the initial and final meson states required by a genuine test of TimeReversal is made by using the pair of time-ordered decays as filtering measurements. Combining the eight independent intensities associated to time-ordered flavour-CP eigenstates of the decay products, BABAR has measured separate asymmetries for each of $\mathrm{CP}, \mathrm{T}, \mathrm{CPT}$ transformations. TRV has been observed, at $14 \sigma$ level, in the time evolution of the neutral B-meson. The measurement of the TRV asymmetries for neutral Kaons in DAPHNE is envisaged.

There are well motivated arguments for a full programme of TRV-Effects in B and K entangled systems.

\section{Acknowledgments}

I would like to thank the Organizers of the Corfu Summer Institute, for the opportunity to discuss these problems in the stimulating atmosphere of the Workshop. This research has been supported by Grants of the Spanish Science Plan with the Project FPA-2011-23596 and of Generalitat Valenciana with the Project GVPROMETEOII 2013-017.

\section{References}

[1] J. H. Christenson, J.W. Cronin, V.L. Fitch and R. Turlay, Phys.Rev 140, B74 (1965)

[2] M. Kobayashi T. and Maskawa, Prog.Theor.Phys. 49, 652 (1973).

[3] BABAR Collaboration, J.L. Lees et al., Phys. Rev. Lett. 109(2012)211801.

[4] M. C. Banuls and J. Bernabeu, Phys.Lett.B 464, 117 (1999)

[5] T. D. Lee and C-N.Yang, Phys.Rev. 104, 254 (1956).

[6] C. S. Wu, E. Ambler, R.W. Hayward, D.D. Hoppes, and R.P. Hudson, Phys.Rev. 105, 1413 (1957).

[7] R.L.Garwin, L.M. Lederman, and M.Weinrich, Phys.Rev. 105, 1415 (1957).

[8] J. H. Christenson, J. W. Cronin, V. L.Fitch, and R. Turlay, Phys.Rev. 140, B74 (1965).

[9] B. Aubert et al. (BABAR Collaboration), Phys.Rev.Lett. 87, 091801 (2001).

[10] K. Abe et al. (Belle Collaboration), Phys.Rev.Lett. 87, 091802 (2001).

[11] N. Cabibbo, Phys.Rev.Lett. 10, 531 (1963).

[12] J. Beringer et al. (Particle Data Group), Phys.Rev.D 86, 010001 (2012).

[13] L. Wolfenstein, Phys.Rev.Lett. 51, 1945 (1983).

[14] M. Antonelli, D. M. Asner, D. A. Bauer, T. G. Becher, M. Beneke, et al., Phys.Rept. 494, 197(2010).

[15] M. Perl et al., Phys.Rev.Lett. 35, 1489 (1975).

[16] S.W. Herb et al., Phys.Rev.Lett. 39, 252 (1977).

[17] B.Aubert et al. (BABAR Collaboration), Nucl.Instrum.Meth.A, 615(2013).

[18] B. Aubert et al. (BABAR Collaboration), Nucl.Instrum.Meth.A 479, 1 (2002).

[19] A. Abashian et al., Nucl.Instrum.Meth.A 479, 117 (2002).

[20] The LHCb Collaboration, JINST 3, S008 005(2008). 
[21] T. Gershon, Y. Nir, in RPP, Chin. Phys. 38, Number 9 (2014).

[22] LHCb Collaboration, R. Aaij et al., Phys. Rev. Lett. 110, 028101 (2013)

[23] J. Bernabeu, M. B. Gavela, A. Santamaria, Phys. Rev. Lett. 57, 1514(1986)

[24] J. Matias, N. Serra, Phys. Rev. D 90, 3, 034002(2014)

[25] BABAR Collaboration, Lees J P, Phys. Rev. D 86 051105(2012)

[26] E. P. Wigner, Nachr. Ges. Wiss. Götingen 32, 35(1932).

[27] L. Wolfenstein, Phys.Rev.Lett. 83, 911 (1999).

[28] L. Wolfenstein, Int.J.Mod.Phys.E 8, 501 (1999).

[29] E. M. Henley, Int.J.Mod.Phys.E 22, 1330010 (2013).

[30] R.G. Sachs, "The Physics of Time Reversal", The University of Chicago Press, 1-325(1987).

[31] R. D. Peccei and H. R. Quinn, Phys.Rev.Lett. 38, 1440 (1977).

[32] J. Beringer et al. (Particle Data Group), Phys.Rev.D 86, 010001(2012).

[33] P. K. Kabir P K, Phys.Rev.D 2, 540 (1970).

[34] A. Angelopoulos et al. (CPLEAR Collaboration), Phys.Lett.B 444, 43 (1998).

[35] V. Weisskopf and E. P. Wigner, Z.Phys. 63, 54 (1930).

[36] W. Weisskopf and Wigner E P, Z.Phys. 65, 18 (1930).

[37] J. P. Lees et al. (BABAR Collaboration), Phys.Rev.Lett. 111,101802 (2013).

[38] E. Nakano et al. (Belle Collaboration), Phys.Rev.D 73, 112002 (2006).

[39] L. Alvarez-Gaume, C. Kounnas, S. Lola, and P. Pavlopoulos, Phys.Lett.B 458, 347 (1999).

[40] J. R. Ellis and N.E. Mavromatos, Phys.Rept. 320, 341 (1999).

[41] H.J.Gerber, Eur.Phys.J.C 35, 195 (2004).

[42] M. C. Banuls and J. Bernabeu, Nucl.Phys.B 590, 19 (2000).

[43] L. Wolfenstein, Int.J.Mod.Phys.E 8, 501 (1999).

[44] H. R. Quinn, J.Phys.Conf.Ser. 171, 011001 (2009).

[45] J. Bernabeu, F. Martinez-Vidal, and P. Villanueva-Perez, JHEP 1208, 064 (2012).

[46] A. Einstein, B. Podolsky, and N. Rosen, Phys.Rev. 47, 777(1935).

[47] M. D. Reid, P. D. Drummond, W. P. Bowen, E. G. Cavalcanti, P. K. Lam, et al., Rev.Mod.Phys. 81, 1727 (2009)

[48] E. Applebaum, A. Efrati, Y. Grossman, Y. Nir, and Y. Soreq, Phys.Rev.D 89, 076011 (2014).

[49] J. Bernabeu, A. Di Domenico, and P. Villanueva-Perez, Nucl.Phys.B 868, 102 (2013).

[50] J. Bernabeu, F. J. Botella, and M. Nebot, Phys.Lett.B 728, 95 (2014). 